PII: S0040-4039(96)01366-4

\title{
REACTIONS OF $\beta$-NITROSTYRENES WITH GRIGNARD REAGENTS
}

\author{
Ching-Fa Yao,* Wen-Chang Chen, and Yu-Mei Lin \\ Department of Chemistry, National Taiwan Normal University \\ 88, Sec. 4, Tingchow Road, Taipei, Taiwan, 11718, R. O. C.
}

\begin{abstract}
Phenyl- $\beta$-nitrostyrene 1a and $\beta$-nitrostyrene 1b react with Grignard reagents to generate hydroximoyl halides 3 or nitrile oxides 4 after workup with ice cold concentrated aqueous $\mathrm{HX}$ acid solution. Carboxylic acids 5 are the only products isolated from 1b and products 3 or 4 are still obtained from 1a when concentrated sulfuric acid solution is used.

Copyright (C) 1996 Elsevier Science Ltd
\end{abstract}

Conjugated nitroalkenes are exceptional Michael acceptors to organometallic reagents. When nitroalkenes are added to Grignard reagents, high yields of nitroalkanes, aci nitro compounds or carbonyl compounds can be obtained. ${ }^{1}$ Nef found that primary or secondary nitro compounds can be hydrolyzed, respectively, to aldehydes or ketones, by treatment of their conjugate bases with sulfuric acid. Primary nitro compounds are converted into carboxylic acids, through the Meyer reaction ${ }^{2}$ when treated with concentrated sulfuric acid. Thus, either the Nef or the Meyer reaction occurs when the conjugate base is added to the strong acid (Scheme I).

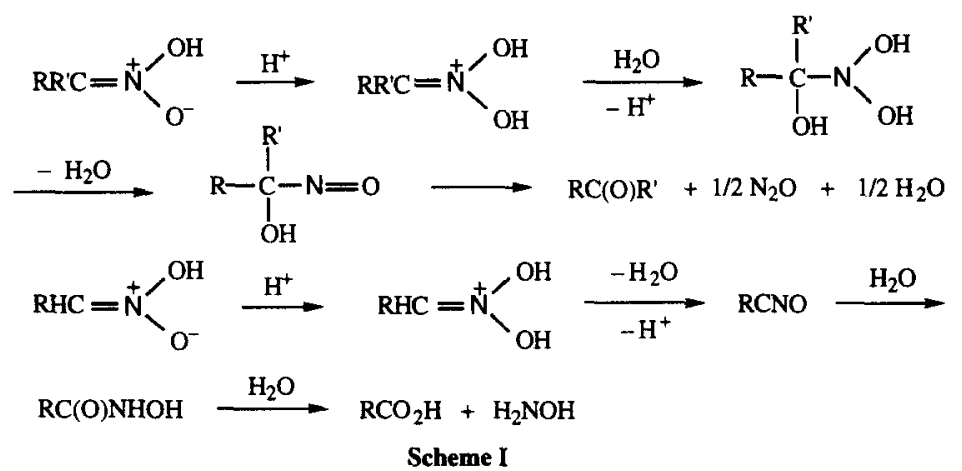

$\alpha$-Phenyl- $\beta$-nitrostyrene 1a and 2-aryl-1-nitroethenes $1 \mathrm{~b}-1 \mathrm{f}$ react with Grignard reagents under a nitrogen atmosphere to give, via 1,4-addition, aci-anions 2 . When aci-anions 2 are added to dilute aqueous hydrochloric acid (5\%) solution, high yields $(60-95 \%)$ of nitroalkanes are obtained. On the other hand, the hydroximoyl halides 3 or nitrile oxides 4 are generated when aci-anions 2 are added to ${ }^{3}$ ice cold concentrated aqueous HX acid solution ( $\mathrm{HCl} 37 \%, \mathrm{HBr} 48 \%$, $\mathrm{HI} 57 \%$ ). However, carboxylic acids 5 are the only products isolated from 2b-2f when $85 \% \mathrm{H}_{2} \mathrm{SO}_{4}$ is used, although compounds 3 and 4 are stable enough to be separated when nitronate $2 \mathbf{a}$ is hydrolyzed in the same condition (Table 1). The generation of different products must be ascribed to steric effect in the aci-anion 2 and the presence of good trapping reagents such as halide. In the case of $2 \mathbf{a}$, the two phenyl groups are presumed to block the attack of the water molecule so that the hydroximoyl halides 3 or nitrile oxides 4 can be isolated. On the other hand, the intermediates 3 and 4 are hydrolyzed to the carboxylic acids 5 
Table 1 The addition of aci-anion 2 to concentrated $\mathrm{HX}_{(\mathrm{mq})}$ or $85 \% \mathrm{H}_{2} \mathrm{SO}_{4(\mathrm{aq})}$

\begin{tabular}{|c|c|c|c|c|c|c|}
\hline \multirow[b]{2}{*}{ Entry } & \multirow[b]{2}{*}{ nitrostyrene } & \multirow[b]{2}{*}{$\mathbf{R M g X}$} & \multirow[b]{2}{*}{$\mathrm{HX}_{(\mathrm{aq})}$} & \multicolumn{3}{|c|}{ (\% yield) } \\
\hline & & & & 3 & 4 & 5 \\
\hline 1 & 1a & $\left(\mathrm{CH}_{3}\right)_{3} \mathrm{CMgCl}$ & $\mathrm{HCl}$ & & 50 & \\
\hline 2 & $1 \mathrm{a}$ & $\left(\mathrm{CH}_{3}\right)_{2} \mathrm{CHM} \mathrm{MCl}$ & $\mathrm{HCl}$ & 77 & & \\
\hline 3 & $1 \mathrm{a}$ & $\mathrm{CH}_{3} \mathrm{CH}_{2} \mathrm{CH}_{2} \mathrm{MgCl}$ & $\mathrm{HCl}$ & 96 & & \\
\hline 4 & $1 \mathrm{a}$ & $\mathrm{CH}_{3} \mathrm{CH}_{2} \mathrm{CH}_{2} \mathrm{MgBr}$ & $\mathrm{HBr}$ & 93 & & \\
\hline 5 & $1 \mathrm{a}$ & $\mathrm{CH}_{3} \mathrm{MgCl}$ & $\mathrm{HCl}$ & 82 & & \\
\hline 6 & $1 \mathrm{a}$ & $\mathrm{CH}_{3} \mathrm{MgBr}$ & $\mathrm{HBr}$ & 85 & & \\
\hline 7 & $1 \mathrm{a}$ & $\mathrm{CH}_{3} \mathrm{MgI}$ & HI & 95 & & \\
\hline 8 & $1 \mathbf{a}$ & $\mathrm{C}_{6} \mathrm{H}_{5} \mathrm{CH}_{2} \mathrm{MgCl}$ & $\mathrm{HCl}$ & 95 & & \\
\hline 9 & la & $\mathrm{C}_{6} \mathrm{H}_{5} \mathrm{CH}_{2} \mathrm{MgCl}$ & $\mathrm{HCl}$ & & $95^{*}$ & \\
\hline 10 & $1 \mathrm{a}$ & $\mathrm{C}_{6} \mathrm{H}_{5} \mathrm{CH}_{2} \mathrm{MgBr}$ & $\mathrm{HBr}$ & 94 & & \\
\hline 11 & $1 \mathrm{a}$ & $c-\mathrm{C}_{6} \mathrm{H}_{11} \mathrm{MgBr}$ & $\mathrm{HBr}$ & & 70 & \\
\hline 12 & $1 b$ & $\left(\mathrm{CH}_{3}\right)_{2} \mathrm{CHMgCl}$ & $\mathrm{HCl}$ & 77 & & \\
\hline 13 & $1 \mathrm{c}$ & $\left(\mathrm{CH}_{3}\right)_{3} \mathrm{CMgCl}$ & $\mathrm{HCl}$ & 56 & & \\
\hline 14 & 1d & $\left(\mathrm{CH}_{3}\right)_{3} \mathrm{CMgCl}$ & $\mathrm{HCl}$ & 56 & & \\
\hline 15 & 1d & $\left(\mathrm{CH}_{3}\right)_{2} \mathrm{CHMgCl}$ & $\mathrm{HCl}$ & 48 & & \\
\hline 16 & 1d & $\mathrm{CH}_{3} \mathrm{CH}_{2} \mathrm{CH}_{2} \mathrm{MgCl}$ & $\mathrm{HCl}$ & 75 & & \\
\hline 17 & 1d & $\mathrm{C}_{6} \mathrm{H}_{5} \mathrm{CH}_{2} \mathrm{MgCl}$ & $\mathrm{HCl}$ & 93 & & \\
\hline 18 & le & $\left(\mathrm{CH}_{3}\right)_{3} \mathrm{CMgCl}$ & $\mathrm{HCl}$ & 30 & & \\
\hline 19 & le & $\left(\mathrm{CH}_{3}\right)_{2} \mathrm{CHMgCl}$ & $\mathrm{HCl}$ & 92 & & \\
\hline 20 & If & $\left(\mathrm{CH}_{3}\right)_{2} \mathrm{CHMgCl}$ & $\mathrm{HCl}$ & 50 & & \\
\hline 21 & la & $\left(\mathrm{CH}_{3}\right)_{3} \mathrm{CMgCl}$ & $\mathrm{H}_{2} \mathrm{SO}_{4}$ & & 62 & \\
\hline 22 & $1 \mathrm{a}$ & $\mathrm{CH}_{3} \mathrm{CH}_{2} \mathrm{CH}_{2} \mathrm{MgBr}$ & $\mathrm{H}_{2} \mathrm{SO}_{4}$ & 89 & & \\
\hline 23 & $1 \mathrm{a}$ & $\mathrm{C}_{6} \mathrm{H}_{5} \mathrm{CH}_{2} \mathrm{MgCl}$ & $\mathrm{H}_{2} \mathrm{SO}_{4}$ & 95 & & \\
\hline 24 & $1 b$ & $\left(\mathrm{CH}_{3}\right)_{3} \mathrm{CMgCl}$ & $\mathrm{H}_{2} \mathrm{SO}_{4}$ & & & 49 \\
\hline 25 & $1 b$ & $\left(\mathrm{CH}_{3}\right)_{2} \mathrm{CHMgBr}$ & $\mathrm{H}_{2} \mathrm{SO}_{4}$ & & & 55 \\
\hline 26 & $1 b$ & $\mathrm{CH}_{3} \mathrm{CH}_{2} \mathrm{CH}_{2} \mathrm{CHMgBr}$ & $\mathrm{H}_{2} \mathrm{SO}_{4}$ & & & 81 \\
\hline 27 & $1 \mathrm{c}$ & $\mathrm{CH}_{3} \mathrm{MgI}$ & $\mathrm{H}_{2} \mathrm{SO}_{4}$ & & & 44 \\
\hline 28 & lf & $\mathrm{C}_{6} \mathrm{H}_{5} \mathrm{CH}_{2} \mathrm{MgCl}$ & $\mathrm{H}_{2} \mathrm{SO}_{4}$ & & & 53 \\
\hline
\end{tabular}

* The $\mathrm{CH}_{2} \mathrm{Cl}_{2}$ extraction is nuetralized by $\mathrm{NaHCO}_{3(\mathrm{aq})}$ solution. 
when 2 b-2f are treated with ice cold $85 \%$ sulfuric acid (Scheme II). Hydroximoyl halides 3 are believed from $\alpha$-nitroso halides $\mathbf{C}$ because the transient intense blue color always is observed when nitronates 2 are added to concentrated acids. $\alpha$-Nitroso halides $\mathrm{C}$ arise if halide (from $\mathrm{RMgX}, \mathrm{MgX}_{2}$ and/or $\mathrm{HX}_{(\mathrm{aq})}$ ) trap the protonated nitronates $\mathbf{A}$, analogous to water in the Nef reaction, or the nitroso cation $\mathbf{B}$. A similar reaction has been reported in which $\alpha$-chloronitroso compounds are formed from nitronate salts using dry hydrogen chloride. ${ }^{4}$ To the best of our knowledge, this is the first example of isolating hydroximoyl halides 3 and nitrile oxides $\mathbf{4}$ from the reactions of nitroalkenes with Grignard reagents.<smiles>[R]C(C)=CCC(C)C</smiles>
1

a: $\mathrm{Ar}=\mathrm{R}^{1}=\mathrm{Ph}$

b: $\mathrm{Ar}=\mathrm{Ph}, \mathrm{R}^{1}=\mathrm{H}$

c: $\mathrm{Ar}=4-\mathrm{FC}_{6} \mathrm{H}_{4}, \mathrm{R}^{1}=\mathrm{H}$

d: Ar=2-thienyl, $\mathbf{R}^{\mathbf{l}}=\mathbf{H}$

e: Ar=2-furyl, $R^{1}=H$

f: Ar=1-naphthyl, $\mathrm{R}^{1}=\mathrm{H}$<smiles>[R]C([R])([Y])C(=O)O</smiles>

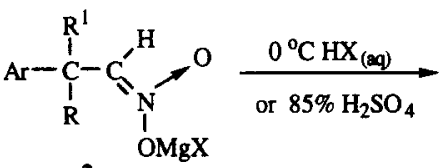

2<smiles>[R]C([Y])([Y])C([N+](=O)[O-])=[N+](O)O</smiles><smiles>[Y]CCCCCC</smiles><smiles>[R]C([R])([Y])C([X])([R])N=O</smiles>

C

Scheme II

It is known that nitrlle oxides 4 can undergo 1,3-dipolar cycloaddition with olefins or acetylenes to generate 2isoxazoline or isoxazole respectively. ${ }^{5}$ The reaction of pure nitrile oxides 4 or hydroximoyl halides 3 in the presence of triethylamine can react with diethyl fumarate, diethyl maleate or phenylacetylene to generate high yields (92-98\%) of additional products. It is also easy to synthesize some intramolecular 1,3-dipolar cycloaddition products when $\beta$-nitrostyrenes ${ }^{6}$ react with proper Grignard reagents (Scheme III).

Acknowledgment. The financial support by the National Science Council of R.O.C. (Grant NSC 83-0208-M003-032) and X-ray data are collected and processed by professor C.-H. Ueng of National Taiwan Normal University are gratefully acknowledged.<smiles>O=CC(Cc1ccccc1)(c1ccccc1)c1ccccc1</smiles><smiles>CCCOCCO</smiles><smiles>CC=CC=CCC</smiles><smiles></smiles><smiles>CCOC(=O)[C@H]1ON=C(C(Cc2ccccc2)(c2ccccc2)c2ccccc2)[C@H]1C(=O)OCC</smiles>

$6(98 \%)$ 


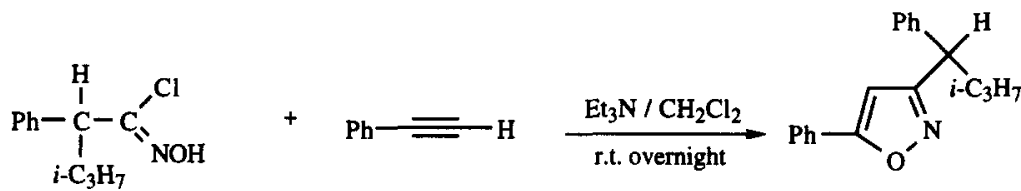

7 (92\%)
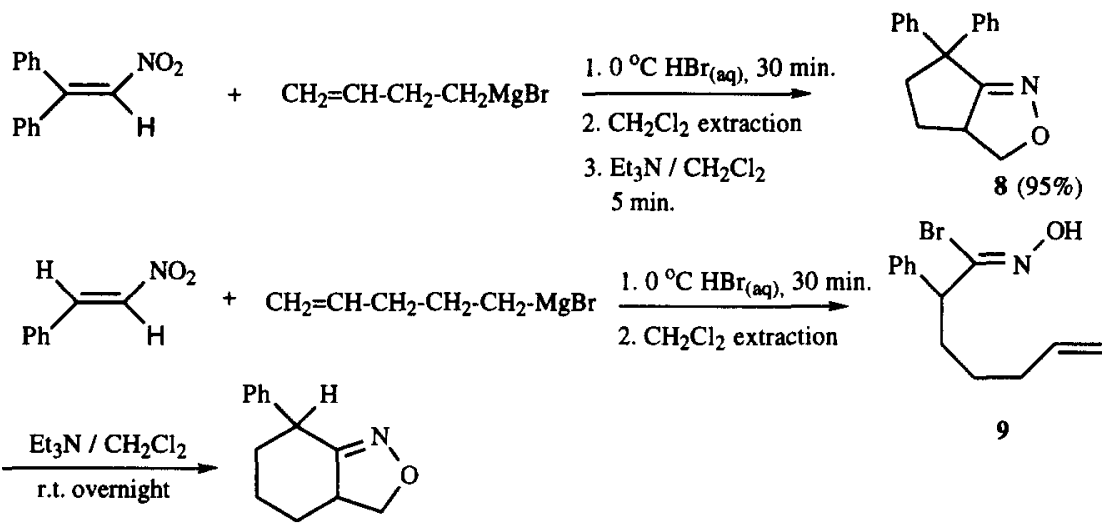

9

$10(88 \%)$

Scheme III

\section{References and Notes}

1. (a) Kohler, E. P.; Stone, J. F. J. Am. Chem. Soc., 1930, 52, 761-768. (b) Buckley, G. D. J. Chem. Soc.,1947, 1494-1497. (c) Ashwood, M. S.; Bell, L. A.; Houghton, P. G.; Wright, S. H. B. Synthesis, 1988, 379-381. (d) Barboni, L.; Bartoli, G.; Marcantoni, E.; Petrini, M.; Dalpozzo, R. Chem. Soc. Perkin Trans 1. 1990, 2133-2138.

2. Pinnick, H. W. Org. React. 1990, 38, 655-792.

3. The general procedure is to add nitroalkene ( $2 \mathrm{mmol}$ ) in $10 \mathrm{~mL}$ THF to $10 \mathrm{mmol}$ of the Grignard reagent in 30 $\mathrm{mL}$ of THF at $-20^{\circ} \mathrm{C}$. Within 10 minutes, the solution is added to ice cold saturated aqueous $\mathrm{HX}(\mathrm{HCl} 37 \%$, $\mathrm{HBr} 48 \%$, $\mathrm{HI} 57 \%$ ) solution and stirred 30 minutes. The solution is extracted with $\mathrm{CH}_{2} \mathrm{Cl}_{2}$, dried over $\mathrm{MgSO}_{4}$, filtered and concentrated to give the products 3 or 4 . The $\mathrm{CH}_{2} \mathrm{Cl}_{2}$ extraction can be neutralized by $\mathrm{NaHCO}_{3(\mathrm{aq})}$ or $\mathrm{Et}_{3} \mathrm{~N}$ to generate nitrile oxides 4 (entry 9) or furoxan (entry 15) after flash column separation in the absence of dipolarophile. The yields are based on NMR by using toluene or diiodomethane as internal standard.

4. Metzger, H.; Meier, H. In "Methoden der Organische Chemie (Houben-Wey-Muller)", Georg Thieme Verlag: Stuttgart, 1971; Vol. X/1, p 979.

5. (a) Wade, P. A.; Amin, N. V.; Yen, H.-K.; Price, D. T.; Huhn, G. F. J. Org. Chem. 1984, 49, 4595-4601.

(b) Torsell, K. B. G. Nitrile Oxides, Nitrones, and Nitronate in Organic Synthesis 1988, VCH, New York.

6. Compound 8: MP $87{ }^{\circ} \mathrm{C} ;{ }^{1} \mathrm{H}-\mathrm{NMR}\left(200 \mathrm{MHz}, \mathrm{CDCl}_{3}\right) \delta 7.40-7.12(\mathrm{~m}, 10 \mathrm{H}), 4.69-4.53(\mathrm{~m}, 1 \mathrm{H}), 4.00-$

$3.78(\mathrm{~m}, 2 \mathrm{H}), 3.11-2.88(\mathrm{~m}, 2 \mathrm{H}), 2.18-2.01(\mathrm{~m}, 1 \mathrm{H}), 1.85-1.67(\mathrm{~m}, 1 \mathrm{H}) ;{ }^{13} \mathrm{C}-\mathrm{NMR}\left(200 \mathrm{MHz}, \mathrm{CDCl}_{3}\right) \delta$

$174.63,144.23,143.66,128.86,128.27,127.61,126.26,127.03,126.64,75.45,53.89,53.67,43.95$,

25.27. Calculated for $\mathrm{C}_{18} \mathrm{H}_{17} \mathrm{NO}$ : C, $82.10 ; \mathrm{H}, 6.51 ; \mathrm{N}, 5.32$. Found: C, $81.99 ; \mathrm{H}, 6.56 ; \mathrm{N}, 5.11$.

(Received in Japan 27 May 1996; revised 8 July 1996; accepted 12 July 1996) 